\title{
Rapid Detection of Biomolecules in a Junction Less Tunnel Field-Effect Transistor (JL-TFET) Biosensor
}

\author{
Rohit Bhargav Peesa ${ }^{1} \cdot$ Deepak Kumar Panda ${ }^{1}$ (D)
}

Received: 21 November 2020 / Accepted: 21 January 2021 / Published online: 4 February 2021

(C) Springer Nature B.V. 2021

\begin{abstract}
In this paper, we present a double gate JL-TFET based biosensor by varying the gate dielectric constant to detect various biomolecules through label-free detection technique. An investigation regarding properties and behavior device has been investigated with the help of Silvaco TCAD. It is observed that Junction less TFET has advantageous over JLFET and TFETdue to the absence of junctions. The proposed device shows reduced short channel effects, lower subthreshold swing along with a higher voltage gain. The detection of the biomolecule is done by varying the gate dielectric constant inside the cavity and different electrical parameters such as electric field, sensing current, threshold voltage and transconductance are observed. The change in the threshold voltage, drain current, transconductance has been used as the sensing metric to detect the sensitivity of the presented device for biomolecule detection. Biomolecule position inside the cavity have been investigated to find out their effect on sensitivity parameter.
\end{abstract}

Keywords Biosensor $\cdot$ Dielectric Permitivity $\cdot$ Junctionless TFET $\cdot$ MOSFET $\cdot$ Subthreshold swing

\section{Introduction}

The present pandemic situation has proved the requirement of biosensors for detecting sars-cov-2(Covid19). Over the last decade, the usage of electrochemical biosensors for the detection of pathogens, DNA, protein, tumour and various other biomolecules has increased. Most of the generic testing techniques have higher false-positive and higher false-negative rates which is a drawback. Moreover, the generic biomolecule testing methodologies are costly, time-taking and involve laborious processes.

There is a need for a sensor to discard the exacting process used in generic testing techniques. A productive sensor is the one which has less error rate, consumes lower power and gives output faster. The detection of biomolecules using a biosensor can be done in two methods either by using Label Based technique or Label-Free detection technique. Label-free detection technique involves detection based on physical properties such as permittivity, sensitivity, selectivity. These

Deepak Kumar Panda

deepakiitkgp04@gmail.com

1 School of Electronics, VIT-AP University, Amaravati, Andhra Pradesh 522237, India physical properties play a good role in affecting the inputoutput characteristics of the device. Since label-free detection techniques do not involve chemical reaction with a biomolecule, it makes them reusable for detecting multiple times.

FET based biosensors are the best devices to implement a label-free detection approach. The demand for FET based sensors has always been increasing as they are swift, unambiguous and accurate. Researchers have developed FET based biosensors even for contagious diseases like Ebola. FET based biosensors are superior as they can be customized to different applications/analytes. They also help us achieve high sensitivity and selectivity for specific biomolecule [1]. FET based biosensors offer ultra-sensitivity, mass production capability and low-cost manufacturing [2].

Despite having many advantages, MOSFET's have a disadvantage: the amount of leakage current in the channel region of MOSFET's increases while decreasing the size of.

the transistor. This increase in the amount of leakage current consequentially increases the power consumption [3]. FET based biosensors have short channel effects in addition to very sharp doping profile [4]. Transistors named tunnel field-effect transistors(TFETs) were introduced to resolve the issue of short channel effects. TFET's could achieve subthreshold swing(SS) below $60 \mathrm{mV} /$ decade which could not be achieved using MOSFET's. Along with these advantages, the 
ability to decrease operating voltage has made them the best alternatives to MOSFETs in low power circuits [5, 6]. Even though TFET's reduced short channel effects and provided superior gate controllability, the issue of doping gradient remains to make it tougher to fabricate devices in nanoscale [7]. Colinge $\mathrm{J}$ et al. introduced the junctionless transistors by defining the physics behind them and their properties [8]. The device has superior properties and is easier to fabricate as it doesn't have any junctions. JLFETs have gained popularity because of their ability to recognize unlabelled molecules faster and lower production cost [9]. JLFETs are highly scalable and highly sensitive while operating over the target biomolecule to be detected.

Junctionless FETs have resolved the hurdles in fabrication. However, they could not overcome the short channel effects, while TFETs could lessen short channel effects. A device named JL-TFET which could provide advantages of both Junctionless transistors and tunnel field-effect transistors was introduced. The reduced the SS along with tunnelling also helps in operating the device at lower voltages. JL-TFET devices work on quantum mechanical principles, specifically band to band tunnelling principle as they are TFET based models [10]. They have to potential to drive high body current due to the absence of a barrier. Increasing the doping or channel thickness increases the drain current of the device [11]. Optimum doping and optimal channel thickness are suggested because a higher doping or increased channel thickness may increase the leakage current.

In this paper, we have presented and analysed the usage of JLTFET as a biosensor for detection of biomolecules. The paper is organized as follows: Section 2 describes the proposed model and device working. Section 3 illustrates the results simulated in Silvaco TCAD. Section 4 is the last section of the paper that concludes our proposal.

\section{Device Structure and Working Principle}

The detection of the biomolecule is through the label-free methodology. Once the biomolecule is absorbed into the nanocavity; the analyte binds the recognition element. The absorbed analyte causes a coupling effect between the gate and channel, thereby affecting the surface charge distribution. The disturbed charge distribution would affect the surface potential and channel conductance. Change in surface potential and channel conductance influences the flow of current between source and drain, it can be measured [12] using an ammeter. The variation in sensing current and threshold voltage proves the presence of biomolecule in the cavity.

Figure 1 shows the proposed device structure for detecting biomolecules. The Si-channel of length $20 \mathrm{~nm}$ and $5 \mathrm{~nm}$ thickness in the proposed model is heavily doped with an n-type material of uniform concentration $1 \times 10^{19} \mathrm{~cm}^{-3}$. The length of control gates and polarity gates is $20 \mathrm{~nm}$ each. This is a lateral model of n-type junctionless TFET having two control gates and two polar gates with different work functions which are isolated by the gap called spacer of length $5 \mathrm{~nm}$ to provide better controllability over the channel.

The proposed model implements the structure proposed in the Design of Double-Gate MOSFET [13], they propose that the introduction of the second gate on the other side of the body increases the scalability. Double gate devices are very advantageous because they have great control over short channel effects [14] which is an issue in sub-50 nm nodes. Double gate devices also reduce subthreshold leakage and increase ON-current because of formation of two conduction channels which makes it more suitable for circuits designed in sub-50 regime [15-18]. We have isolated the gates because creating isolation in between gates reduces power consumption [19, 20]. The model contains a high- $\mathrm{K}$ gate dielectric which is TiO2. DG-TFET with high-K gate dielectric [21] proposes a novel design of DG-TFET with the higher gate dielectric. They propose that subthreshold slope for fixed gate bias remains constant and exhibits smaller threshold voltage shift, unlike the devices with $\mathrm{SiO} 2$.

The presence of two types of the gates above Si channel layer enables the conversion of the highly doped $n^{+}-n^{+}-n^{+}$ substrate region to $\mathrm{p}^{+}-\mathrm{i}-\mathrm{n}^{+}$substrate region in the drain, channel and source respectively without doping. The gates have different work functions each of $4.3 \mathrm{eV}$ and $5.93 \mathrm{eV}$ respectively for Control-gate and P-gate. Different work functions for Control-gate and P-gate give improvised results [22] which is a lower subthreshold slope and higher Ion/Ioff ratio. Simulations and results of the device have been obtained by using Silvaco Atlas, 2D Device Simulation ver-5.15.32.R. We have used the non-local band to band tunnelling model available in Silvaco Atlas [23].

Non-local band to band tunnelling model has been used because it provides more precise result than the local band to band tunnelling model $[24,25]$. Local band to band models are disadvantageous because they either assume maximum electric field or average electric field while a non-local model assumes it to be dynamic at each point in the path and tunnelling current is calculated at each mesh point, making the current depend on the band edge profile along the complete path. Additionally, we have used the newton method [23] to solve the equations. Its capability to converge faster irrespective of the size of the problem provokes us to use newton method. Moreover, it is the default method to solve coupled equations in Atlas [23].

The proposed JLFET biosensor is recommended when compared to conventional FET based biosensor because they can be fabricated even at lower costs. After all, the doping concentrations and type of doping is the same for source and drain which is not possible in conventional FET biosensor. JLFET biosensors consume lower power than conventional 
Fig. 1 2D structure of proposed JL-TFET for Biomolecule Detection. The width of the device is $1 \mu \mathrm{m}$

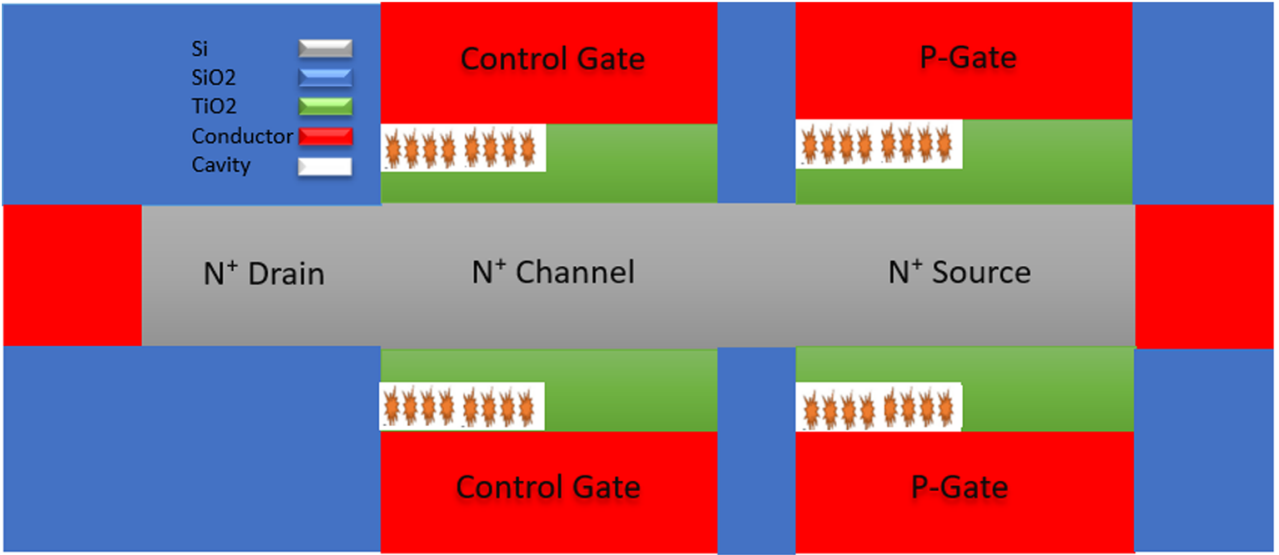

FET biosensors. This biosensor has higher performance and less power loss because of having higher Ion/Ioff ratio. Furthermore, it is resistant to dopant fluctuations as it doesn't have dopant atoms.

\section{Results and Discussions}

\subsection{Device Characteristics}

Figure 2 shows the energy band diagram of the device along the $\mathrm{x}$-coordinate; when the gate bias is applied to the control gate and the polarity gate remains at zero bias. The diagram shows that the bandgap between the valence band and the conduction band is low. The lower bandgap shows that there is a higher probability of electrons to tunnel from valence band to conduction band, consequently increasing the current flow. The device exhibits a similar electric field for each case when the device is tested for different biomolecules.

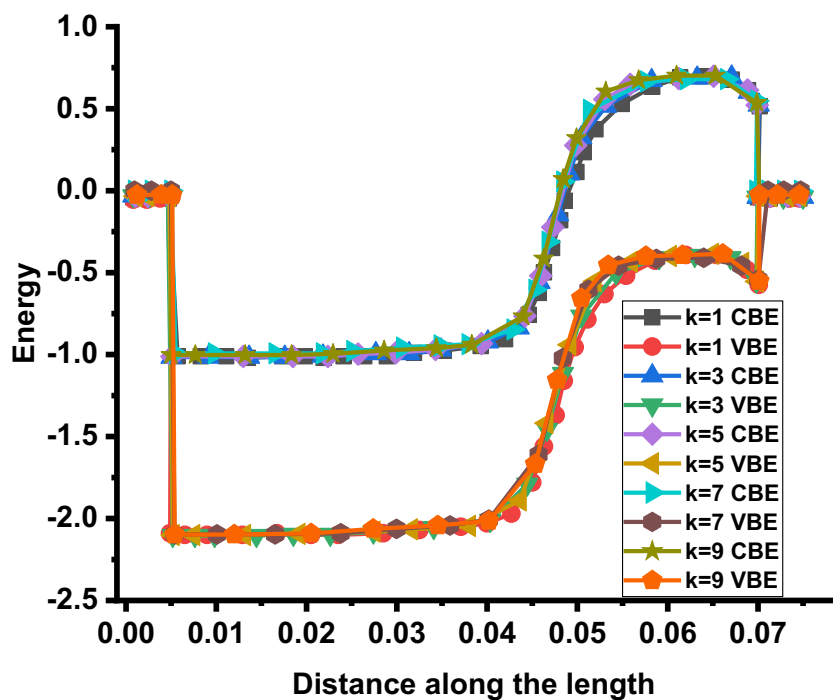

Fig. 2 Energy band diagram from source to drain along the length of the device
Figure 3 shows the distribution of the electric field along the length of the device. We observe that the electric field is smaller in the drain region, later increases to approximately $2.25 \times 10^{5} \mathrm{~V} / \mathrm{cm}$ at the juncture of the channel and source; soon decreases in the drain region. A higher value at juncture is because electric field's strength increases as more space charge moves towards juncture, causing a maximum accumulation at that point.

Figure 4. illustrates the way the potential of the device varies along the length of the device. The potential of the device is approximately $1 \mathrm{~V}$ in drain region which instantaneously rises to $1.5 \mathrm{~V}$ in the channel region but later falls to $0 \mathrm{~V}$ in the source region.

\subsection{Input Characteristics}

Figure 5 depicts the transfer characteristics of the proposed model for various biomolecules with values of permittivity as above. It can be observed that as the permittivity of the biomolecule increases; the device's subthreshold region decreases. Drain current is highest i.e.0.241 $\mu \mathrm{A},-6.61798$ (log Scale) for biomolecule with $\mathrm{K}=9$, least $13.5 \mathrm{nA}$, $-7.96816(\log$ Scale) for $\mathrm{K}=1$ which is air and for other $\mathrm{K}$

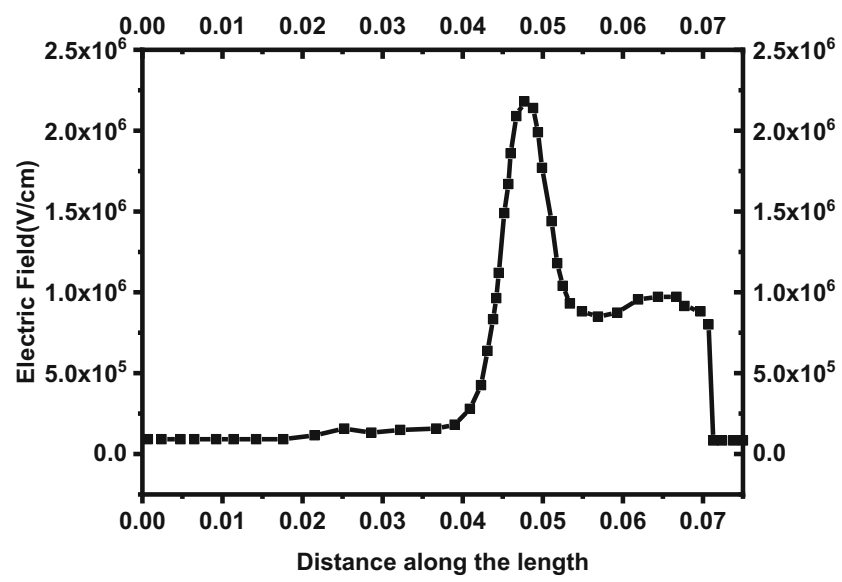

Fig. 3 Electric field from source to drain along the length of the device 


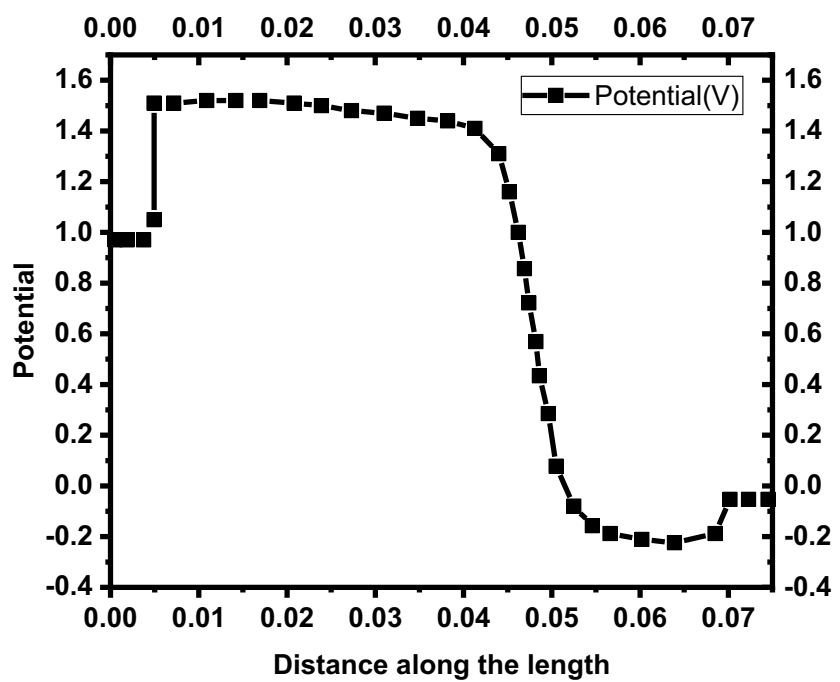

Fig. 4 Channel Potential(V) from source to drain along the length of the device

values it keeps increasing with $\mathrm{K}$ value. It helps us infer that the drain current in $\mathrm{ON}$-state also increases as the permittivity of biomolecules increases.

Figure 6 depicts the ratio of Ion/Ioff for different biomolecules of different $\mathrm{K}$ values. The device could attain exceptional Ion/Ioff ratio $=6 \times 10^{8}$ for biomolecule with permittivity $=$ 7. Permittivity 7 is the average permittivity of the outer shell of human protein. A higher Ion/Ioff is required because it indicates better performance and lower power loss through leakages. Higher Ion/Ioff provides more gate controllability which helps in faster switching.

The threshold voltage is an important parameter which shows the minimum voltage flowing from gate to source [26]. Figure 7 shows that there is a decrease in threshold voltage $(\mathrm{Vt})$ with an increase in the permittivity value of biomolecule. Fig. 7 shows the variation in the sensitivity of the device

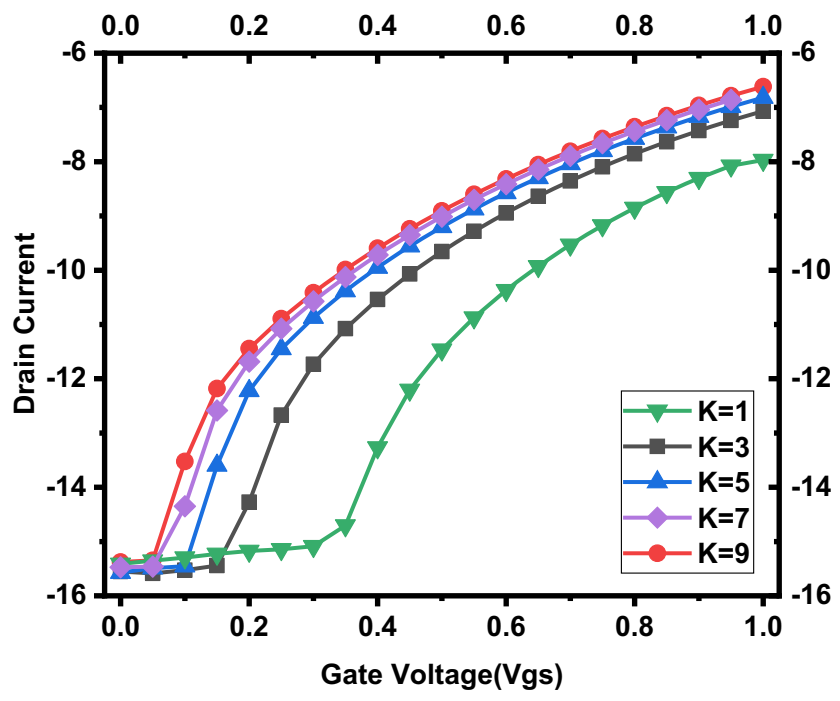

Fig. 5 Transfer Characteristics for different biomolecules

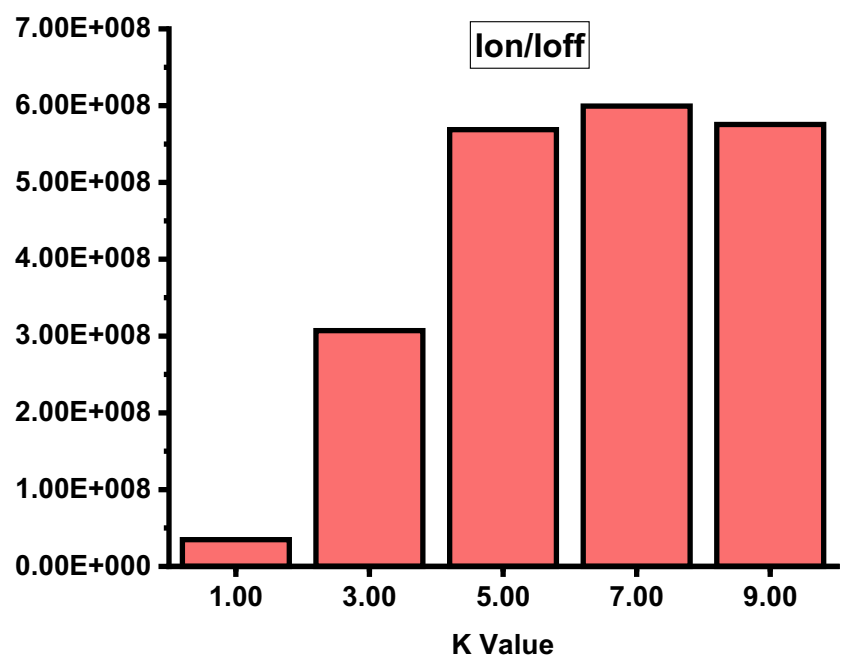

Fig. 6 Ion/Ioff for different $\mathrm{K}$ values

for different biomolecules. It can be seen that sensitivity for $\mathrm{K}=1$ is 0 because the sensitivity of a device is considered by comparing it with the working of the device for air whose $\mathrm{K}$ value is 1 .

Sensitivty $=\frac{\frac{\text { Ion }}{\text { Ioff }}(\text { bio })-\frac{\text { Ion }}{\text { Ioff }}(\text { air })}{\frac{\text { Ion }}{\text { Ioff }}(\text { bio })}$

Furthermore, subthreshold slope (SS) is another important feature which shows the behavior of the FET in the subthreshold region. It shows how the drain current behavior is controlled by the gate terminal.

$\mathrm{SS}=\frac{\mathrm{d} V_{g}}{\mathrm{~d} \Psi_{s}} \times \frac{\mathrm{d} V_{s}}{\mathrm{~d}\left(\log I_{\mathrm{ds}}\right)}, \frac{d V_{g}}{\mathrm{~d} \Psi_{s}}=\left(1+\frac{C_{s}}{C_{\mathrm{ins}}}\right)$

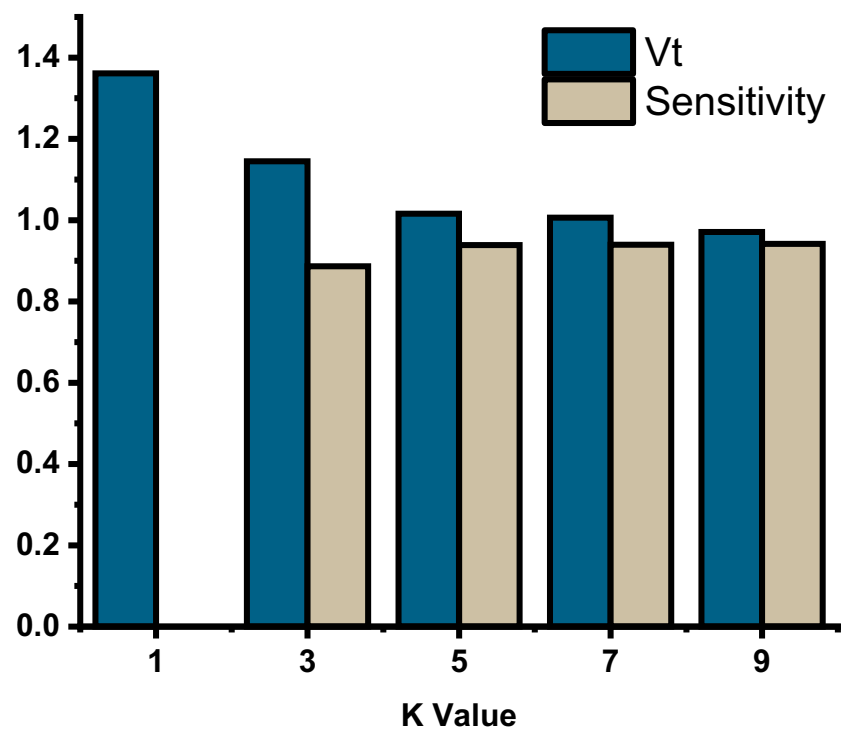

Fig. 7 Bar plot for Vt and sensitivity 
Figure 8 illustrates a line plot with the subthreshold slope in $\mathrm{mV} /$ Decade on the $y$-axis and permittivity of the biomolecule on the x-axis. As the permittivity of the biomolecule increases; the device shows a peculiar trend which is subthreshold slope can neither be considered as directly proportional to permittivity nor be considered as inversely proportional to permittivity. Nevertheless, it serves its purpose of attaining a SS value less than $70 \mathrm{mV} /$ decade which cannot be achieved by using a conventional MOSFET.

Transconductance(gm) is another major input characteristic which helps in evaluating the performance of Analog Device. Transconductance parameter can be calculated by differentiating transfer characteristics [27].

$\mathrm{gm}=\mathrm{dIds} / \mathrm{dVgs}$

Figure 9 is a plot depicting the variation of transconductance(gm) with gate voltage (Vgs) for various values of biomolecules. The plot depicts that when we increase the gate voltage; as the value of permittivity of biomolecule increases the transconductance of the device also increases.

\subsection{Comparison Based on Filling}

Figure 10 illustrates the transfer characteristics of the device when different cavities are filled with biomolecules. It can be observed that the behaviour of the device when all cavities are filled with protein and only cavities under P-gate are filled with protein are almost similar, while the device behaviour when cavities under control gates filled with protein, all cavities filled with air, only cavity under P-gate or only cavity under control gate exhibit similar characteristics after the threshold region that is after

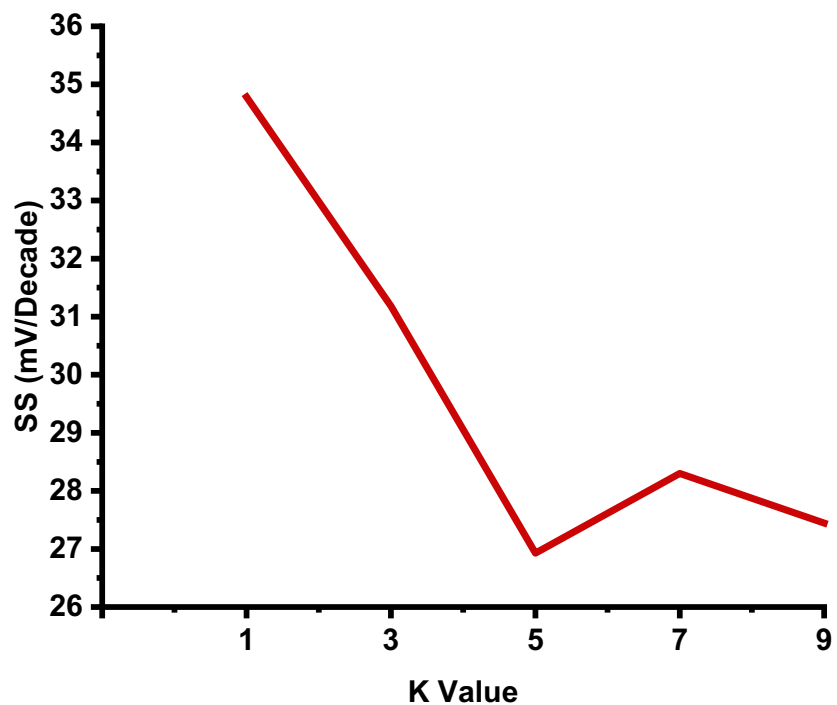

Fig. 8 Line plot showing for Subthreshold Slope vs K- Value

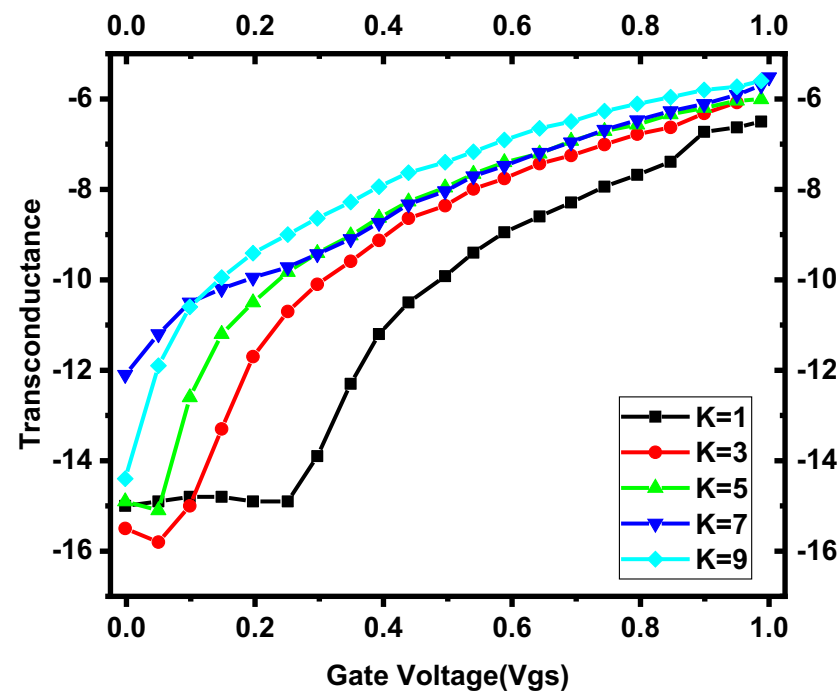

Fig. 9 Transconductance vs gate voltage

$0.39 \mathrm{~V}$. Control gates only help in controlling the $\mathrm{ON}$ and OFF states of the device with lower semiconductor losses, while on the contrary $\mathrm{P}$ gates help in the conduction of current. A positive $\mathrm{P}$ gate helps in current flow through electrons, while a negative $\mathrm{P}$ gate helps in current flow through holes [28]. This capability of P gate makes cavity below $\mathrm{P}$ gate a major affecter. Hence, similar properties are observed when the $\mathrm{P}$ gate is filled and all gates are filled.

Table 1 depicts the variations in threshold voltage, subthreshold slope and transconductance after filling different types of cavities. Despite exhibiting similar characteristics when few types of cavities are filled; threshold voltage, subthreshold slope and transconductance values vary for each condition.

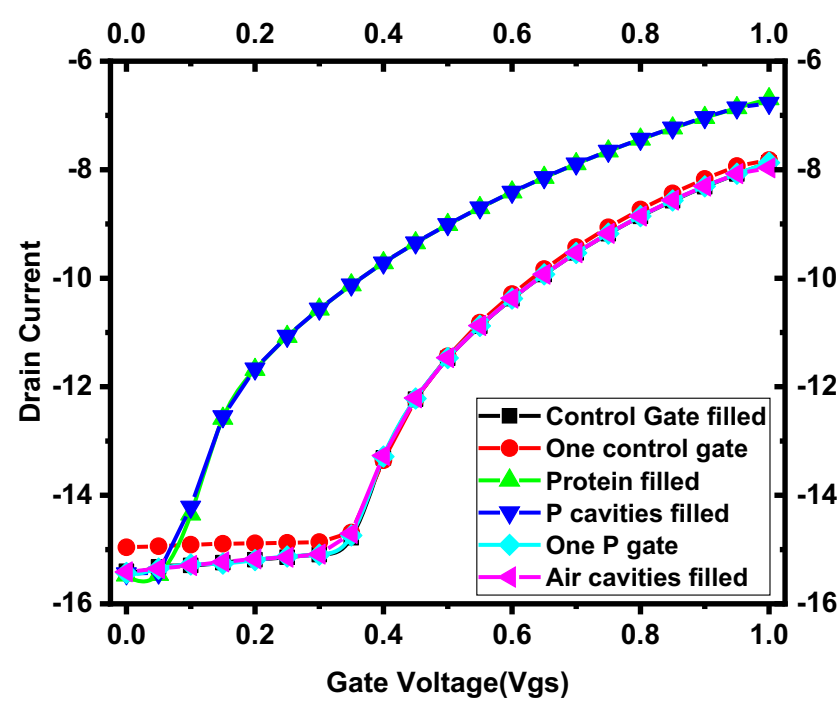

Fig. 10 Transfer characteristics for different cavities 
Table 1 Variation of Vt, SS and gm for different cavity fillings

\begin{tabular}{llll}
\hline Filling & Vt & SS & Gm \\
\hline All four cavities-Protein & $1.00617 \mathrm{~V}$ & $27.4463 \mathrm{mV} / \mathrm{dec}$ ade & $1.73358 \mathrm{e}-016$ \\
All four cavities-Air & $1.3614 \mathrm{~V}$ & $34.7827 \mathrm{mV} / \mathrm{dec}$ & $9.64958 \mathrm{e}-016$ \\
Control gates with protein and polarity gates with Air & $1.34948 \mathrm{~V}$ & $33.7132 \mathrm{mV} / \mathrm{dec}$ & $1.09237 \mathrm{e}-015$ \\
Polarity gates with protein and control gates with Air & $0.987123 \mathrm{~V}$ & $32.8035 \mathrm{mV} / \mathrm{dec}$ & $9.16928 \mathrm{e}-013$ \\
Once control gate is filled with protein & $1.35717 \mathrm{~V}$ & $34.4061 \mathrm{mV} / \mathrm{dec}$ & $3.80135 \mathrm{e}-016$ \\
One polar gate is filled with protein & $1.40155 \mathrm{~V}$ & $37.5557 \mathrm{mV} / \mathrm{dec}$ & $4.8039 \mathrm{e}-016$ \\
\hline
\end{tabular}

\section{Conclusion}

In this paper, we proposed the implementation of JL-TFET as a biosensor for the detection of various biomolecules. The device is designed and calibrated in Silvaco TCAD tool. Detection of the biomolecule is done by observing variations in various sensing parameters such as threshold voltage, sensing current, potential and transconductance. From the results, we conclude that the proposed JL-TFET model can act as a superior biosensor for biomolecule detection in comparison to existing FET based biosensors for low power application. The position of biomolecules inside the nanogap cavity have been varied to find out their effects on sensitivity. It has been concluded that maximum sensitivity could be obtained when all of the cavity regions are filled with a biomolecule.

Acknowledgements The authors acknowledge VIT-AP university for providing necessary facilities for carrying out the research work.

Availability of Data and Material Not applicable.

Code Availability Not applicable.

\section{Declaration}

Conflict of Interest The authors declare that they have no conflict of interest.

Compliance with Ethical Standards The manuscript is prepared by as per the ethical standard of the journal.

\section{Consent to Participate Not applicable.}

Consent for Publication The authors have given Consent for Publication as per the journal policy.

\section{References}

1. Mao S, Lu G, Yu K, Bo Z, Chen J (2010) Specific protein detection using thermally reduced Graphene oxide sheet decorated with gold nanoparticle-antibody conjugates. Adv 22:3521-3526. https://doi. org/10.1002/adma.201000520

2. Syu Y-C, Hsu W-E, Lin C-T (2018) Review-Field-Effect Transistor Biosensing: Devices and Clinical Applications. ECS J Solid State Sci Technol 7:Q3196-Q3207

3. Kao K et al (2012). IEEE Trans Electron Devices 69:2070-2077
4. Im H, Huang XJ, Gu B, Choi YK (2007) A dielectric-modulated field-effect transistor for biosensing. Nature Nanotech 2:430-434

5. Aghandeh H, Ziabari SAS (2017) Gate engineered heterostructure junctionless TFET with Gaussian doping profile for ambipolar suppression and electrical performance improvement. Superlattices Microstruct. https://doi.org/10.1016/j.spmi.2017.06.018

6. Ionescu M, Riel H (2011) Tunnel field-effect transistors as energyefficient electronic switches. Nature 479:329-337

7. Agarwal A, Pradhan PC, Swain (2019) B.P. effects of the physical parameter on gate all around FET. Sādhanā. https://doi.org/10. 1007/s12046-019-1232-8

8. Colinge JP et al (2011) In: Nazarov A, Colinge JP, Balestra F, Raskin JP, Gamiz F, Lysenko V (eds) semiconductor-on-insulator materials for Nanoelectronics applications. Springer, Berlin

9. Kim CH, Jung C, Lee KB, Park HG, Choi YK (2011) Label-free DNA detection with a nanogap embedded complementary metaloxide-semiconductor. Nanotechnology 22:135502. https://doi.org/ 10.1088/0957-4484/22/13/135502

10. Niranjan Reddy K, JayaSree PVY (2016) Tunnel Field Effect Transistor- Most Promising Device- Review on TFET. IJEECS 4(9): $1-5$

11. Twinkal Solankia, Nilesh Parmar (2001) A Review paper: A Comprehensive study of Junctionless transistor https://www. bvmengineering.ac.in/misc/docs/published-20papers/etel/etel/ 401011.pdf Accessed 2001

12. Colinge JP. (2014) Silicon-on-insulator (SOI) junctionless transistors Manufacturing and Applications 167-194 https://doi.org/10. 1533/9780857099259.1.167

13. Srivastava V.M., Singh G. (2014) Design of Double-Gate MOSFET. In: MOSFET Technologies for Double-Pole FourThrow Radio-Frequency Switch. Analog Circuits and Signal Processing, vol 122. Springer https://doi.org/10.1007/978-3-31901165-3 3

14. Chang L, Yang C, Ha D, Ramade P, Xiong S, Bokor J, Hu C, Tsu K (2003) Extremely scaled silicon nano CMOS devices. Proc IEEE. https://doi.org/10.1109/JPROC.2003.818336

15. Wong HSP, Frank DJ, Solomon PM (1998) Device design considerations for double-gate, ground-plane, single-gated ultra-thin SOI MOSFET at the $25 \mathrm{~nm}$ channel length generation Proceedings of Int. Electron Devices Meet. https://doi.org/10.1109/IEDM.1998. 746385

16. Nowak E, Aller I, Ludwig T, Kim K, Bernstein K (2004) Turning silicon on its edge [double gate CMOS/FinFET technology]. IEEE Circuits Device Magazine. https://doi.org/10.1109/MCD.2004. 1263404

17. Tang S, Chang L, Lindert N, Xuejue H, Bokor J, Chenming H (2001) FinFET-a quasi-planar double-gate MOSFET. Proc IEEE Int Solid-State Circuits Conf. https://doi.org/10.1109/ISSCC.2001. 912568

18. Fried DM, Nowak EJ, Kedzierski J, Duster JS, Komegay KT (2003) A Fin-type independent-double-gate NFET. Proc Device Res Conf. https://doi.org/10.1109/DRC.2003.1226864 
19. Mukhopadhyay S, Mahmoodi H, Roy K (2004) High performance and low power domino logic using independent gate control in double-gate SOI MOSFETs. Proc of IEEE Int SOI Conf. https:// doi.org/10.1109/SOI.2004.1391558

20. Mukhopadhyay S, Mahmoodi H, Roy K (2005) Design of highperformance sense amplifier using independent gate control in sub$50 \mathrm{~nm}$ double-gate MOSFET. Proc 6th Int Symp Qual Electronic Design. https://doi.org/10.1109/ISQED.2005.44

21. Boucart K, Ionescu AM (2007) Double-Gate Tunnel FET With High-K Gate Dielectric. IEEE Trans Electron Devices. https://doi. org/10.1109/TED.2007.899389

22. Ghosh B, Akram MW (2013) Junctionless Tunnel Field Effect Transistor. IEEE Electron Device Lett. https://doi.org/10.1109/ LED.2013.2253752

23. ATHENA/ATLAS User's Manual (2012) SILVACO International: Santa Clara http://statistics.roma2.infn.it/ messi/SIC/sic_21-01-04/ atlas98-v1_users.pdf Accessed November 1998

24. Biswas A, Dan SS, Le Royer C, Grabinski W, Ionescu AM (2012) TCAD simulation of SOI TFETs and calibration of non-local bandto-band tunneling model. Microelectron Eng 98:334-337

25. Hussain S et al (2020) A comprehensive study on tunneling field effect transistor using non-local band-to-band tunneling model. J
Phys: Conf Series. https://doi.org/10.1088/1742-6596/1432/1/ 012028

26. Das R, Baishya S (2019) Analytical modelling of electrical parameters and the analogue performance of cylindrical gate-all-around FinFET. Pramana - J Phys. https://doi.org/10.1007/s12043-0181663-5

27. Cao W, Yao CJ, Jiao GF, Huang D, Yu HY, Li MF (2011) Improvement in reliability of tunneling field-effect transistor with pnin structure. IEEE trans. Electron Devices 58:2122-2126

28. De Marchi M et al (2012) Polarity control in double-gate, gate-allaround vertically stacked silicon nanowire FETs. Int Electron Devices Meet. https://doi.org/10.1109/IEDM.2012.6479004

Publisher's Note Springer Nature remains neutral with regard to jurisdictional claims in published maps and institutional affiliations. 